\title{
LA EDUCACIÓN EPISTOLAR: LOS INTERCAMBIOS DE CARTAS ENTRE MUJERES BURGUESAS COMO FUENTE DE DESARROLLO PERSONAL EN LA INGLATERRA VICTORIANA
}

\author{
Meritxell Simon-Martin ${ }^{1}$
}

\section{RESUMEN}

Este artículo examina la correspondencia personal de tres mujeres inglesas burguesas, Anna Mary Howitt, Barbara Leigh Smith Bodichon y Bessie Rayner Parkes, como fuentes de desarrollo personal. A partir del concepto de 'educación epistolar' (SIMON-MARTIN, 2016, 2016a, 2020), este artículo explora la carta como instrumento educativo en el sentido del término neohumanista alemán Bildung (formación a lo largo de la vida), y sugiere que estas tres amigas no sólo reflexionaron sobre temas como el amor y el matrimonio a través de las cartas sino también concertaron acuerdos con sus pretendientes y familiares que respectaran sus necesidades matrimoniales y sus expectativas profesionales. Paralelamente, este articulo incluye una breve reflexión epistemológica sobre cómo abordar la cuestión del 'silencio archivístico', dado que se basa en gran medida en el análisis de la voz epistolar de Bessie únicamente.

Palabras clave: Bildung, formación, cartas, educación epistolar, silencio archivístico.

\footnotetext{
${ }^{1}$ University of Roehampton (UR), Londres, Reino Unido.
} 


\title{
A EDUCAÇÃO EPISTOLAR: OS INTERCÂMBIOS DE CARTAS ENTRE MULHERES BURGUESAS COMO FUENTES DE DESARROLLO PESSOAL NA INGLATERRA VITORIANA
}

\section{RESUMO}

Este artigo examina a correspondência pessoal de três mulheres burguesas inglesas, Anna Mary Howitt, Barbara Leigh Smith Bodichon e Bessie Rayner Parkes, como fontes de desenvolvimento pessoal. Com base no conceito de 'educação epistolar' (SIMON-MARTIN, 2016, 2016a, 2020), este artigo explora a carta como um instrumento educacional no sentido do termo neo-humanista alemão Bildung (aprendizagem ao longo da vida) e sugere que esses três amigas não apenas refletiram sobre amor e casamento através das cartas, mas também concluíram acordos com seus pretendentes e familiares que respeitavam suas necessidades conjugais e expectativas profissionais. Ao mesmo tempo, este artigo inclui uma reflexão epistemológica sobre como abordar a questão do 'silêncio arquivístico', uma vez que se baseia amplamente na análise da voz epistolar de Bessie.

Keywords: Bildung, formação, cartas, educação epistolar, silêncio arquivístico.

\section{EPISTOLARY EDUCATION: LETTER EXCHANGES AMONG VICTORIAN MIDDLE-CLASS WOMEN AS SOURCES OF SELF-DEVELOPMENT}

\begin{abstract}
This article examines the personal correspondence of three Victorian English women, Anna Mary Howitt, Barbara Leigh Smith Bodichon and Bessie Rayner Parkes as sources of selfdevelopment. Drawing on the concept of 'epistolary education' (SIMON-MARTIN, 2016, 2016a, 2020), it explores the letter as an educational instrument - education understood in the sense of the German neo-humanist concept Bildung (the lifelong process of self-development). It suggests that these three Friends not only discussed themes such as love and marriage via letters but also reached agreements with their suitors and family that respected their matrimonial needs and professional expectations. Given that it is underpinned by an analysis of the epistolary voice of Bessie almost exclusively, this article also includes an epistemological reflection upon how to tackle the question of 'archival silence'.
\end{abstract}

Key words: Bildung, self-development, letters, epistolary education, archival silence.

\section{L'ÉDUCATION ÉPISTOLAIRE : LES ÉCHANGES DE LETTRES ENTRE FEMMES BOURGEOISES EN TANT QUE SOURCES DE FORMATION PERSONNELLE DANS L'ANGLATERRE VICTORIENNE}

\section{RESUME}

Cet article vise à étudier la correspondance personnelle de trois femmes bourgeoises, Anna Mary Howitt, Barbara Leigh Smith Bodichon et Bessie Rayner Parkes, en tant que sources de 
développement personnel. A partir du concept d'éducation épistolaire' (SIMON-MARTIN, 2016, 2016a, 2020), cet article explorera la lettre en tant qu'instrument éducatif dans le sens du terme néo-humaniste allemand Bildung (la formation tout au long de la vie). L'article suggère que ces trois amies ont non seulement réflexionné sur l'amour et le mariage au moyen des lettres mais aussi ont accordé, avec leurs prétendants et leurs familles, des consensus qui respecteraient leurs besoins matrimoniaux et leurs expectatives professionnelles. Parallèlement, cet article inclut une brève réflexion épistémologique sur la façon d'aborder la question du 'silence archivistique', étant donné qu'il se base majoritairement sur l'analyse de la voix épistolaire de Bessie.

Mots-clés: Bildung, formation personnelle, lettres, éducation épistolaire, silence archivistique. 


\section{INTRODUCIÓN}

Anna Mary Howitt (1824-1884), Barbara Leigh Smith Bodichon (18271891) y Bessie Rayner Parkes (1829-1925) fueron tres mujeres que vivieron durante el apogeo industrial, económico y colonial de Gran Bretaña. Se conocieron a mediados de los años 1840 en Hastings, en el sur de Inglaterra, cuando tenían entre 17 y 22 años, y mantuvieron la amistad hasta el final de sus vidas. Su correspondencia personal, dispersa en varios archivos británicos y estadunidenses, dan testimonio de la amistad que forjaron y mantuvieron en el marco de su estilo de vida 'nómada'. Y es que, siguiendo la moda burguesa de la Inglaterra victoriana, alternaban, en función de la época del año, estancias en sus diferentes casas (en Londres, Hastings, Cornwall, Birmingham, etc) con visitas a casa de familiares y amigos y viajes de turismo y de convalecencia. Además, casadas con un médico y un pintor franceses respectivamente, Barbara y Bessie vivieron a caballo entre Inglaterra, Argelia y Francia durante años.

En el contexto de este estilo de vida nómada y holgado, la correspondencia permitió a estas tres amigas estar en contacto no sólo entre ellas sino también con su familia y con sus amigos y conocidos allá donde viajaran. El acto de leer, escribir y enviar cartas era un gesto que formaba parte de su cotidiano. Estos intercambios epistolares, más allá de su vocación comunicativa principal, funcionaban como un medio psicológico y social: actuaban como un espacio de expresión emocional e intelectual y forjaban relaciones interpersonales. Además, el acto de leer y escribir cartas también sirvió para intercambiar, adquirir y producir conocimiento, ejercer el razonamiento, desarrollar el espíritu crítico, en definitiva, articular y forjar su subjetividad, no solamente entre ellas durante su adolescencia sino con otros destinatarios a lo largo de su vida.

Como ya he escrito en otras publicaciones, el término educativo neohumanista alemán Bildung (generalmente traducido en español como formación a lo largo de la vida) ofrece un marco conceptual para examinar los intercambios epistolares como plataforma dialógica de aprendizaje y desarrollo 
personal (SIMON-MARTIN, 2016, 2016a, 2020). En el marco de esta lectura de la carta como instrumento educativo (como fuente de Bildung), en este artículo me gustaría centrarme en una dimensión del desarrollo personal que las tres amigas discutieron en detalle: el amor, y más en concreto, el matrimonio. Dado el estado de los archivos personales de estas tres amigas, me gustaría además hacer una pequeña reflexión epistemológica sobre la carta como fuente de conocimiento histórico. $\mathrm{Y}$ es que los archivos personales de estas tres mujeres albergan muchas cartas escritas por Bessie, pero sólo un número relativamente limitado de cartas escritas por Barbara y Anna Mary. A partir de las cartas que escribió Bessie y en menor medida Anna Mary, en este artículo voy a examinar la carta como fuente de razonamiento epistolar.

En su libro Becoming a Woman in the Age of Letters, Dena Goodman describe el rol de los intercambios epistolares en la vida de las mujeres burguesas francesas de finales del siglo XVIII. Goodman explica cómo aprendían el arte de escribir epístolas por medio de manuales, secretarios y conversaciones epistolares con damas de la corte. Goodman sugiere el término razonamiento epistolar ("epistolary reasoning”) para describir el uso que estas mujeres hacían de la carta para intercambiar consideraciones sobre el amor, las peticiones de matrimonio, el noviazgo y el matrimonio (GOODMAN, 2009, p. 277). En línea con la propuesta de Goodman, en este artículo voy a explorar en qué medida Bessie, Barbara y Anna Mary no sólo reflexionaron sobre el amor y el matrimonio a través de las cartas sino también concertaron acuerdos con sus pretendientes y familiares que respectaran sus necesidades matrimoniales y sus expectativas profesionales. En términos de Bildung, voy a ilustrar como los diálogos epistolares sirvieron de espacio (1) donde negociaron y ejercieron su autonomía (la piedra angular de este concepto educativo), y, de forma concomitante, (2) donde articularon una redefinición de los conceptos de feminidad, amor y matrimonio.

En efecto, Bildung es un concepto filosófico neohumanista alemán que emergió durante la Ilustración, c.1750-1830. En su definición general, el 
término hace referencia al proyecto educativo, a lo largo de la vida, de convertirse en un individuo instruido (gebildet) con el objetivo de contribuir, de forma colectiva, al progreso de la humanidad. A diferencia de la mera adquisición de una habilidad manual mecánica, Bildung aspira a fomentar el potencial individual formando a les personas intelectual, moral y físicamente. Este desarrollo personal a lo largo de la vida tiene lugar en interacción con el mundo externo. El hombre cultivado tiene una relación recíproca pero crítica con su entorno - en un proceso de mimética reflexiva. El mundo externo permite al hombre acercarse a todo aquello que le resulta desconocido y éste lo incorpora en su "yo" de una forma analítica. Para conseguir esta conexión crítica con el exterior, el hombre tiene que distanciarse de sí mismo y de sus creencias. El aprendizaje crítico y el subsecuente desarrollo personal que resulta tienen lugar siempre y cuando el hombre adopte una actitud abierta hacia el nuevo conocimiento y las perspectivas de otras personas (HUMBOLDT, c.1793-1794; véase también BLEICHER, 2006; BRUFORD, 1975; LØVLIE, MORTENSEN y NORDENBO, 2003; LÜTH, 1998; VARKØY, 2010; WIMMER, 2003; WULF, 2003).

Las relaciones sociales son precisamente el medio a través del cual se puede adquirir la riqueza del otro. Las semejanzas y las diferencias entre un individuo y las personas que le rodean crean una fricción que propicia el desarrollo de su subjetividad. En este sentido, según Bildung, los viajes ofrecen una oportunidad excelente para exponerse a las semejanzas y a las diferencias que emanan de la diversidad del mundo (HUMBOLDT, 1796-1797). En el epicentro de esta concepción del proceso de desarrollo personal a lo largo de la vida se encuentra el principio de autonomía. Bildung se basa en el supuesto de que la racionalidad humana permite al hombre evaluar la realidad de forma crítica y actuar en consecuencia, siempre y cuando no haya ni coacción ni manipulación.

Bildung es pues un concepto educativo que busca desarrollar la individualidad a partir de la interacción social. Es a través de las relaciones con 
otras personas, especialmente durante los viajes, que el hombre se expone a la diferencia y la incorpora de forma crítica en su subjetividad, para después poder ejercer su autonomía y, en última instancia, contribuir como ciudadano al desarrollo de la sociedad. En base a esta concepción de la educación, lo que sugiero es que los intercambios epistolares reflejan la esencia de Bildung: el proceso intersubjetivo de desarrollo personal a lo largo de la vida. Es a partir de la comunicación epistolar con otras personas que Anna Mary, Barbara y Bessie, no sólo mantuvieron una interacción recíproca transformativa entre ellas (mimética creativa), sino que además pasaron por las fases educativas que implica Bildung: la adquisición de conocimiento, el distanciamiento con uno mismo (inclusive propiciado por los viajes) y el ejercicio de la autonomía. En otras palabras, paralelamente al acto de comunicarse con familiares, amigos y conocidos, en sus cartas las tres amigas, al menos parcialmente, adquirieron formación intelectual, se forjaron una subjetividad como resultado de la fricción con su entorno, y ejercieron su autonomía (SIMON-MARTIN, 2012, 2016, 2016a, 2020).

A continuación voy a explorar el rol de la carta en el proceso de razonamiento epistolar en relación al tema del amor y al matrimonio a partir de dos grupos de conversaciones epistolares: las cartas que Anna Mary, Bessie y Barbara se intercambiaron entre ellas a raíz de la lectura de un poema, donde acabaron abordando la cuestión de los derechos de las mujeres; y las cartas que Bessie intercambió con sus pretendientes y familiares, donde pactó las condiciones de su noviazgo y su futura vida matrimonial. Paralelo a este análisis de la Bildung epistolar de estas tres amigas, mi estudio propondrá una reflexión epistemológica introductoria sobre cómo abordar la cuestión de los silencios archivísticos, dado que mi argumento se basa en gran medida en el análisis de la voz epistolar de Bessie únicamente.

Con el objetivo de contribuir a los debates epistemológicos actuales sobre la producción del conocimiento histórico a partir de fuentes primarias autobiográficas (autobiografías, memorias, diarios, cartas, blogs, cuentas de 
Instagram, etc), este artículo ilustra la relevancia del concepto de relacionalidad narrativa de Sidonie Smith y Julia Watson: es decir, el hecho de que el 'yo' autobiográfico no hable de forma aislada sino en relación a otras personas y en referencia a las vidas y a la realidad de éstas, lo que implica que los textos autobiográficos de un autor incorporan, intrínsecamente, relatos sobre terceras personas (SMITH y WATSON, 2001, p. 94-96). A partir del concepto de relacionalidad narrativa, la primera sección de este artículo sugiere que si se presta atención a los relatos sobre un sujeto histórico que están presentes en las cartas que le fueron enviadas y en las que hablan de ella/él en tercera persona es decir, respectivamente a su "yo" epistolar en las cartas que recibió y a su "ella/él" epistolar en las cartas que hacen referencia a ella/él - permite hacer "hablar" a los silencios que los archivos personales de este sujeto histórico ha dejado, en este caso, con el objetivo de poder estudiar la manera en que Barbara y Anna Mary ejercieron su autonomía en relación con una dimensión del desarrollo personal: el amor y el matrimonio.

\section{"EL MATRIMONIO O ALGÚN TIPO DE UNIÓN ENTRE HOMBRES Y MUJERES ES NUESTRO DESTINO"}

Filántropa, artista y una de las impulsoras del movimiento a favor de los derechos de las mujeres en Inglaterra, Barbara nació en el seno de una familia Unitaria, muy progresista y acomodada. Fue la hermana mayor de los cinco hijos ilegítimos de un hombre de negocios y diputado del Partido Liberal. Su madre, sombrerera, falleció de tuberculosis cuando Barbara cumplió 7 años. Ella y sus hermanos vivieron entre las dos casas que poseía el padre: una en Londres y otra en Hastings. Los cinco hermanos recibieron una educación informal en casa, donde aprendieron historia, literatura, geografía, política económica, latín y pintura, de la mano de varios tutores. El elemento más sorprendente de este amplio plan de estudios fue que Barbara tuvo un acceso no restringido al saber - en forma de clases estimulantes con los tutores; en forma 
de lectura de libros de la biblioteca familiar y de los periódicos a los que su padre estaba suscrito; $y$ en forma de actividades recreativas y sociales, como los salones literarios y políticos que éste organizaba en casa (donde invitaba a las personalidades relevantes del momento), las excursiones en el campo que hacían todos juntos con sus tutores, las sesiones de pintura que hacía en el exterior con su profesor o con amigas, los viajes por Gran Bretaña y en el extranjero que hacía con su familia o con amigas, y por último, el patrón de socialización que seguía: por ejemplo asistiendo a actos culturales, fiestas, charlas públicas y visitando escuelas filantrópicas. (HIRSCH, 1998, p. 18-19).

Su amiga Bessie también nació en el seno de una familia Unitaria de clase media y se convirtió en una de las pioneras del movimiento feminista inglés. ${ }^{2}$ Su padre era abogado y su madre, ama de casa, descendiente del científico Joseph Priestley (al que se le atribuye el descubrimiento del oxígeno). A diferencia de Barbara, Bessie fue escolarizada en una reputada escuela para niñas en Leam, cerca de Birmingham, donde aprendió literatura inglesa, aritmética, historia, francés, y alemán, y, con un tutor particular, latín y griego. Siguiendo la carrera profesional del padre, la familia se mudó a Londres cuando Bessie era todavía una niña y, durante años, intercalaron largas estancias en Hastings, que tenía un clima más temperado y menos contaminado que Londres, a razón de los problemas de salud de su hermano, que murió de tuberculosis siendo aún adolescente. Aunque en un contexto familiar más heterodoxo, Bessie también se benefició de la vida social que su familia le ofrecía: asistiendo a eventos culturales, políticos y filantrópicos, una experiencia educativa que le sirvió para desarrollar su carrera como escritora, periodista y

\footnotetext{
2 Los términos "feminism" y "feminist", derivados del vocablo féminisme en francés, no fueron acuñados en lengua inglesa, por lo menos en Gran Bretaña, hasta finales de la década de 1890 (RENDALL, 1985, p. 39). Los términos que utilizaban las protagonistas de este estudio y sus coetáneas eran "woman's rights woman" para referirse a ellas mismas y "the (woman's) movement", "the cause" o "the woman question" para referirse a sus campañas. En este sentido, se trata de dos términos anacrónicos en este estudio. A pesar de este anacronismo, siguiendo a la historiadora Kathryn Gleadle, utilizo los términos "feminismo" y "feminista" en reconocimiento de los puntos en común que comparten los términos utilizados en el siglo XIX y en la actualidad: una conciencia de la posición subordinada de la mujer en el seno de la sociedad y un activismo enfocado a luchar contra esta discriminación (GLEADLE, 1995, p. 5).
} 
poetisa (SHATTOCK, 2004).

Por su parte, Anna Mary fue la hija mayor del matrimonio de los escritores William y Mary Howitt, muy famosos en aquella época por sus cuentos infantiles, poemas, traducciones y periódicos literarios. Anna Mary nació en Nottingham, pero creció en Esher, en el condado de Surrey. Dado que nació en un entorno familiar literario y en el seno de una familia cuáquera, donde las mujeres, igual que en las familias Unitarias, recibían una educación más sólida que en otras denominaciones religiosas, como los anglicanos o los evangelistas, lo más probable es que tuviera una buena educación en casa, de la mano de sus padres. Mostrando un talento artístico desde la infancia, Anna Mary estudió en Henry Sass' Art Academy, la escuela en la que los estudiantes (varones) se formaban antes de examinarse para entrar en la prestigiosa Royal Academy (que excluía a las mujeres). Es en esta reputada escuela de formación que Anna Mary conoció a los famosos pintores prerrafaelistas Dante Gabriel Rossetti y Holman Hunt. Además, durante dos años, Anna Mary se mudó a Múnich para trabajar bajo la tuición del muralista e ilustrador de libros alemán Wilhelm von Kaulbach (HIRSCH, 1998, p. 21-22, 43-45).

Como ya he explicado en otras publicaciones, durante la adolescencia y a lo largo de su vida, las tres amigas combinaban estancias juntas, donde compartían sus actividades educativas (clases particulares, actividades de ocio, y eventos sociales), artísticas (sesiones de dibujo y pintura), filantrópicas (sobre todo visitas a colegios) y feministas (escribiendo artículos de prensa y panfletos en pro de los derechos educativos, profesionales legales y políticos de las mujeres), y temporadas en las que vivían separadas y se carteaban. En línea con la idea de interacción social formativa de Bildung como medio para forjar la individualidad, las cartas actuaron como una fuente de intercambio de conocimiento, de diálogo con puntos de vista diferentes, y de expresión de la subjetividad. De este modo, las cartas ofrecían a las tres amigas la oportunidad de compartir sus ideas y opiniones sobre los temas y las lecturas que estudiaban con sus tutores en casa y, más allá de la adolescencia, sobre las experiencias 
personales que vivían en el marco de su estilo de vida "nómada". Siguiendo el código epistolar de reciprocidad, se prestaban libros y periódicos, se recomendaban lecturas y acontecimientos sociales, y discutían temas de actualidad como las novedades literarias, la política, la economía, y los problemas sociales, temas que intercalaban con asuntos como la salud, el tiempo, anécdotas divertidas, vicisitudes personales y expresiones de amor y amistad. Estas conversaciones epistolares, entabladas no sólo entre ellas sino con otros miembros de su círculo social, estaban repletas de preguntas dirigidas al recipiente, desencadenando así reacciones que incitaban a responder para expresar las impresiones personales que estas preguntas y estos temas les suscitaban. De este modo, expresando sus opiniones personales en las cartas entrecruzadas, no sólo durante la adolescencia sino a lo largo de la edad adulta, contribuyó a la formación de su individualidad. Y es que las tres amigas verbalizaban sus ideas en el gesto de escribir una carta y, a su vez, en el gesto de contrastar puntos de vista y de recibir feedback, desarrollaron sus opiniones siguiendo un patrón circular, potencialmente infinito (SIMON-MARTIN, 2012, 2016, 2016a, 2020).

En el marco de esta "educación epistolar”, Anna Mary, Barbara y Bessie abordaron en sus cartas una cuestión relevante en sus vidas: el amor y el matrimonio. Según la correspondencia que ha sobrevivido, no es hasta relativamente tarde que las tres amigas discutieron estos temas en sus cartas. Ocupadas como estaban leyendo, asistiendo a charlas, visitando museos, yendo a conciertos de música, publicando artículos en la prensa local, viajando y realizando actividades filantrópicas, lo último que sus cartas ilustran son pensamientos de vida matrimonial. Las obligaciones maritales tradicionales hubieran puesto punto y final en gran medida a estas actividades de ocio y de realización personal. Además, a los 27 años Barbara publicó un panfleto, titulado Brief Summary of the Most Important Laws Concerning Women, donde resumía las leyes matrimoniales de Inglaterra en aquella época, que como bien explicaba, otorgaban plena entidad legal a las mujeres solteras, pero les arrebata de sus derechos de propiedad, de ingresos, de sus hijos y de su 
cuerpo una vez se convertían en esposas (BODICHON, 1854). Habiendo estudiado minuciosamente el sistema legal inglés, el matrimonio hubiera supuesto una pérdida sustancial de libertad personal y legal que ninguna de las tres amigas estaba dispuesta a sufrir. Aunque más tarde de lo que uno hubiera esperado (suponiendo que no abordaran el tema del matrimonio en cartas ahora desaparecidas), las tres amigas utilizaron los intercambios epistolares para expresar y compartir sus pensamientos sobre el amor y el matrimonio a lo largo de las diferentes fases de la vida.

A modo de ejemplo, a finales de 1847 y principios de 1848, Barbara, Bessie y Anna Mary se escribieron una serie de cartas sobre el poema de Alfred Tennyson The Princess, un poema narrativo que explica la historia de una princesa, Ida, a la que comprometen a casarse con un príncipe. Desafiando a su familia, Ida decide, en vez de casarse, fundar una universidad para mujeres. Con la ayuda de dos amigos, el príncipe (disfrazado de mujer) consigue entrar en el edificio de la universidad, pero los tres chicos son descubiertos y, en la lucha por conseguir llevarse a la princesa, son heridos. Las estudiantes de la universidad los curan y, finalmente, Ida decide irse con el príncipe.

La primera carta de la que se tiene constancia fecha del 26 de diciembre de 1847, en la que Anna Mary escribe a Barbara:

Tengo ganas de que llegue el nuevo poema de Tennyson. Me gustaría que llegara esta semana y a la vez no quiero porque no podría resistirme a leerlo y tengo tantas otras cosas que me he propuesto hacer (Columbia University, Butler Library, Leonore Beaky, Letters of Anna Mary to Barbara, letter 4)

Anna Mary lee el libro y, tres días después, el 29 de diciembre, vuelve a escribir a Barbara para recomendarle el libro y compartir con ella sus impresiones: 
encantados. Me gustaría que lo pudieras conseguir, que lo leyeras y que me dieras tu opinión. Creo que le tendría que gustar a todas las mujeres. En cuanto a la conclusión del poema, es preciosa y noble. ... Es un poema sobre la educación de las mujeres, los derechos de les mujeres, la verdadera humanidad de las mujeres y creemos que es noble y verdadero (Columbia University, Butler Library, Leonore Beaky, Letters of Anna Mary to Barbara, letter 5).

El mismo 29 de diciembre, su otra amiga, Bessie, escribe a Barbara una carta lamentándose de no poder comprar una copia del poema:

Todavía no he leído el poema 'The Princess' porque el año pasado me compré más libros de los que debía y he empezado éste con el propósito de contenerme; así que he decidido no comprármelo por ahora (London Metropolitan University, Women's Library, Paparas of Barbara McCrimmon related to Barbara Bodichon, $7 \mathrm{BMC} / \mathrm{F} 16)$.

Unos días más tarde, Bessie vuelve a escribir otra carta a Barbara diciéndole:

He leído Tennyson. Mi madre me lo dio. ¿Como puedo enviártelo? La crítica que hace Anna Mary es excelente. Es verdad que es una mezcla de aspiraciones modernas y de comportamientos caballerosos y es por lo que no será demasiado criticado, pero es un sueño precioso y estoy segura de que te gustará. Ida es a la vez tan audaz y tan silenciosa y majestuosa. Representa el ideal de mujer. El poema es, como dice Anna Mary, "noble y verdadero". Creo que te casarías con el príncipe si existiera una persona así. Estaría incluso a la altura de tu ideal romántico (Cambridge University, Girton College Archives, Girton College Personal Papers (GCPP) Bessie 5/19)

En esta segunda carta que Bessie envía a Barbara descubrimos que Bessie sabe que Anna Mary ha leído el poema y también sabe qué opinión le merece. Lo que no sabemos es si Anna Mary, además de escribir a Barbara, también compartió su opinión sobre el poema con Bessie directamente, o si fue Barbara la que le escribió una carta a Bessie hablándole sobre la opinión de Anna Mary o si le reenvió a Bessie la carta que había recibido de Anna Mary. El 
caso es que Bessie tiene constancia de la opinión de Anna Mary y que las tres amigas están al corriente de lo que dice y piensa cada una.

A finales de enero Bessie escribe una tercera carta a Barbara y concluye:

No encuentras nada absurdo en Ida. Yo en cambio, aunque encontré que era una persona magnífica, estoy de acuerdo con la moralidad de Tennyson. Creo que Ida deja de lado las leyes de Dios cuando intenta poner remedio a la maldad social. Admiro profundamente a Ida por su entusiasmo a favor de las mujeres, pero comete el error de irse al otro extremo y poner a los hombres en una situación inferior en vez de igual a las mujeres. Estoy completamente convencida, como seguro que tú también lo estas, de que el matrimonio o algún tipo de unión entre hombres y mujeres es nuestro destino. Sería una locura negarlo. Tiene que haber una continuación de la especie humana. El objetivo no es prohibir el matrimonio cuando se habla de mejorar la educación y la legislación sino de hacerlo más noble y digno, y una fuente de alegría para las dos partes. Y a mí me da la impresión de que Ida se olvida de eso (GCPP Bessie 5/20)

Lo que esta conversación epistolar fragmentada nos sugiere es que los intercambios epistolares permitieron a Anna Mary, Barbara y Bessie compartir conocimiento y ejercer su pensamiento crítico: les permitió (1) estar al corriente de una nueva publicación, el poema The Princess, (2) recomendarse la lectura, (3) obtener una copia del ejemplar en el caso de que fuera necesario, y (4) desarrollar sus opiniones. El ejercicio del pensamiento crítico funcionó de la siguiente manera, presente también en otras conversaciones epistolares del mismo tipo. La conversación epistolar se inició verbalizando las primeras impresiones y formulando preguntas directas dirigidas a la destinataria. Estas primeras impresiones y preguntas incitaron a la reflexión y a la articulación del pensamiento de la destinataria, que, en su carta-respuesta, contrastó puntos de vista y ofreció feedback. Siguiendo el código epistolar de reciprocidad, se crearon así reacciones en cadena, potencialmente infinitas. En esta cadena de intercambios de impresiones, el gesto de expresar sus ideas y de recibir el feedback de cada una, permitió a estas tres amigas desarrollar su propia opinión sobre el poema y, a través de esta opinión, de perfilar e individualizar su 
subjetividad.

En línea con la idea de Bildung de interacción social formativa como medio para forjar la individualidad, estos fragmentos ilustran la idea de que interactuar con las perspectivas de los otros induce al aprendizaje: comunicarse implica adquirir la riqueza del otro; pero, utilizando la mimética reflexiva, esta interacción con el otro resulta en nuestra propia opinión personal y nuestro propio punto de vista. La fricción que emerge como resultado de contrastar las ideas de los otros conduce a definir cada vez con más precisión la individualidad de cada uno. En este caso, los intercambios de puntos de vista sobre The Princess ofrecieron a estas tres chicas la oportunidad de reflexionar sobre el contenido del poema, hecho que acabó activando una discusión sobre la educación de les mujeres, la legislación y el matrimonio.

Las cartas que Barbara escribió de mano propia se han perdido; pero este diálogo epistolar, aunque sea fragmentado, sugiere que las tres amigas estaban parcialmente en desacuerdo sobre cómo interpretar la acción de Ida, es decir, que cada una verbalizó su propia individualidad. Las tres amigas diferían ligeramente sobre cómo interpretar las hazañas de la princesa Ida. Emocionada con el poema y ansiosa de saber qué opinaba Barbara de él, Anna Mary y su familia estaban "encantados" con un poema que juzgaron "noble y verdadero ... sobre la educación de las mujeres, los derechos de les mujeres, la verdadera humanidad de las mujeres”, y cuya conclusión era "preciosa y noble”. Según Bessie, a Barbara le gustaba el poema y no veía nada particularmente "absurdo en Ida”. Al parecer, Barbara (y quizá también Anna Mary) aprobaba el deseo de Ida de remediar una injusticia en contra de las mujeres con la fundación de una universidad y no veía ninguna contradicción en que Ida combinara este proyecto con su deseo de casarse con el príncipe. Por su parte, Bessie estaba de acuerdo con Anna Mary en que el poema, "una mezcla de aspiraciones modernas y de comportamientos caballerosos", era "noble y verdadero".

Como Barbara, Bessie también pensaba que Ida era "una persona magnífica” y apoyaba que ésta intentara "poner remedio a la maldad social". Sin 
embargo, Bessie discrepaba de Barbara en cuanto que ella sí estaba completamente "de acuerdo con la moralidad de Tennyson", es decir, que, en el proceso, Ida "comete el error de irse al otro extremo y poner a los hombres en una situación inferior en vez de igual a las mujeres”. A los ojos de Bessie, el deseo de Ida de casarse con el príncipe era comprensible porque estaba "completamente convencida" de que "el matrimonio o algún tipo de unión entre hombres y mujeres es nuestro destino". Según ella, "[t]iene que haber una continuación de la especie humana”. El problema es que, según Bessie, Ida, al poner los hombres por debajo de las mujeres, no podía convertir el matrimonio en algo "noble y digno, y una fuente de alegría para las dos partes”.

En definitiva, estos fragmentos sugieren que, como instrumento educativo, el acto de escribir cartas y el gesto cotidiano de intercambiar correspondencia sirvió como un espacio donde las tres amigas adquirieron y compartieron conocimiento y ejercieron y desarrollaron su capacidad de crítrica. Desafiando expectativas normativas de género, en sus cartas presentaron y desarrollaron su propia conceptualización de la feminidad y de la igualdad. En este sentido, las tres amigas ejercieron su autonomía, entendida según la definición feminista de Diana Meyers, como la actuación en armonía con uno mismo (MEYERS, 1989; véase SIMON-MARTIN, 2016, 2020 para una explicación detallada de una lectura crítica de Bildung, incluida la cuestión de la autonomía). Y llegamos a esta conclusión en gran medida gracias a la voz de Bessie. En otras palabras, en esta primera sección he analizado el razonamiento epistolar de estas tres amigas a través del "yo" epistolar de Bessie y de Anna Mary, del "tú" epistolar de Barbara, y del "ella” epistolar de Anna Mary (para una explicación detallada de la diferencia entre estas tres fuentes de información histórica, véase SIMON-MARTIN, 2020). Recurrir a estas fuentes de información epistolar son una de las estrategias a las que los historiadores pueden recurrir para hacer frente a los silencios archivísticos, en este caso el de la voz de Barbara, totalmente ausente en esta conversación epistolar. En efecto, como he explicado en otras publicaciones, existen diferentes estrategias para hacer frente a los silencios archivísticos: entre otras, se puede leer entre líneas o 
recurrir el concepto de relacionalidad narrativa, en el caso concreto de las cartas, recurriendo al "yo" y al "él/ella" epistolar del sujeto "silenciado" (SIMON-MARTIN, 2020, 2020a). En la sección siguiente me centraré en recurrir a la hipótesis a partir del "yo" epistolar de Bessie como estrategia para hacer frente al silencio archivístico.

\section{"ESTOY SUMAMENTE CONTENTA DE QUE APRUEBES MI 'DESCARO FEMENINO JUVENIL'"'}

Barbara tuvo tres peticiones de matrimonio. Su primer pretendiente fue su propio tutor, Philip Kingsford, que se interesó en ella cuando tenía 22 años. El mismo año, 1849, recibió una petición de matrimonio de la parte de James Joseph Sylvester, profesor de matemáticas en la Universidad de Londres. Un año más tarde, Joseph Neuberg, un hombre de negocios de Nottingham, también mostró una atención especial para con ella (HIRSCH, 1998, p. 105). No sabemos en qué condiciones, pero Barbara rechazó estas tres peticiones. El hombre del que, al parecer, se enamoró primero fue de John Chapman, editor del periódico liberal Westminster Review. Los dos se conocían desde hacía años y, durante el verano del 1855, cuando Bodichon tenía 28 años, proyectaron la posibilidad de formar una unión libre, que fue abortada por el padre de ésta (HIRSCH, 1998, p. 106-114). No fue hasta finales de 1856 que Barbara conoció al que fue su marido, Eugène Bodichon, doctor de nacionalidad francesa afincado en Argelia, con el que se casó en Londres, a la edad de 30 años y con el que no tuvo hijos (HIRSCH, 1998, p. 122-129).

En cuanto a Anna Mary, sabemos que, durante su estancia en Múnich a principios de los años 1850, es decir, cuando tenía 27-28 años, estuvo comprometida con Edward Bateman, un ilustrador y decorador de interiores, amigo del grupo de pintores prerrafaelitas. El compromiso se rompió en circunstancias no elucidadas cuando éste emigró a Australia al cabo de unos años de noviazgo (HIRSCH, 1998, p. 45-48). Anna Mary se acabó casando, seis 
años más tarde, con un amigo de infancia, Alfred Watts, en 1859, con el que no tuvo hijos (HIRSCH, 1998, p. 169). Como en el caso de Barbara, no conocemos las condiciones bajo las que Anna Mary aceptó la petición de matrimonio de los dos pretendientes que sabemos que tuvo, ni como acordó los términos del noviazgo; lo único que sabemos es que Anna Mary decidió estudiar durante dos años en Múnich con una amiga mientras su primer prometido se quedó en Inglaterra.

En este sentido, a diferencia de Bessie, los archivos personales de sus dos amigas son muy limitados. Ya sea fruto del azar o de la manipulación (de ellas mismas o familiares, amigos, archiveros, biógrafos/as), sus archivos personales no nos permiten dilucidar cómo Anna Mary y Barbara ejercieron su autonomía en referencia a las peticiones de matrimonio que recibieron, ni a las condiciones de su noviazgo y futuro status como esposas. Para compensar este silencio archivístico irreparable, propongo, ya no recurrir al "yo", "tú" y "ella" epistolar (como he hecho en la sección precedente para hacer "hablar" al silencio que el "yo" epistolar de Barbara ha dejado en la conversación sobre Tennyson), sino recurrir a la hipótesis a partir del “yo” epistolar de Bessie.

Como hemos visto, el poema de Tennyson puso en marcha una conversación intelectual entre Anna Mary, Barbara y Bessie que desencadenó una discusión sobre la educación de las mujeres y el matrimonio. Del mismo modo, otros diálogos epistolares muestran que las tres amigas desarrollaron su propia opinión sobre el amor y el lugar que éste ocupaba en sus vidas durante su adolescencia. Y lo hicieron criticando y burlándose de la normatividad de género (SIMON-MARTIN, 2020). Sin embargo, los archivos personales de Barbara y Anna Mary no nos aportan el tipo de información sobre su perspectiva en el amor y el matrimonio como mujeres casaderas como lo hace el archivo personal de Bessie. En esta sección voy a examinar el ejercicio del razonamiento epistolar exclusivamente a través de las cartas de Bessie y a continuación propondré una hipótesis sobre el de Anna Mary y Barbara.

El 24 de noviembre de 1849 Bessie, con 20 años, recibió una carta con 
una proposición de matrimonio de Robert Fane, un joven escocés al que había conocido un tiempo antes. Al día siguiente de recibir la carta y sin consultar a sus padres, Bessie le respondió a Robert:

Estimado señor,

Su carta, sencilla y directa, merece una respuesta sencilla y directa, y estoy segura de que la apreciará más que una respuesta convencional. Con su petición de matrimonio le hace a una mujer el mayor cumplido que un hombre puede hacerle, y la imposibilidad de colmar su deseo no impide que así lo sienta.

Señor, en el estado actual de la sociedad, una mujer debe, en gran medida, dejarse absorber por la vida y los proyectos de su marido. Para ello es necesario que ésta le conozca profundamente, y que tenga la absoluta certeza de que se podrá consagrar tanto a él como a sus proyectos. Ella deberá ser su devota compañera, no sólo a través de su amor sino a través de su conciencia y su intelecto.

Más allá de su carácter justo y de los méritos que creo que ha logrado, no conozco absolutamente nada de usted; y creo que es más que probable que en mí, una joven chica de veinte años, encontraría miles de defectos de los que ahora no es consciente.

Créame, necesito más un profesor que un marido y espero que continuará siendo el amigo que creí encontrar en usted.

Sinceramente suya, señor,

Bessie Rayner Parkes (GCPP Parkes 9/109)

El mismo día, Bessie envió una carta a su padre, Joseph Parkes, informándole de la situación y de la decisión que ella misma había tomado:

Mi Madre le informará con detalle sobre el hecho de que ayer por la tarde, mientras estaba tranquilamente cosiendo una enagua de franela, recibí una proposición de matrimonio de la mano y corazón del Señor Robert Fane. Me reí a carcajadas, tanto que mi Madre pensó que me había vuelto histérica. Nunca en mi vida había oído algo tan absurdo; ... Me aferro a la satisfacción que me procura, querido Papá, lo que espero será, durante mucho tiempo, mi estilo de vida actual, con [John Stuart] Mill, latín, montar a caballo, las críticas literarias, [Thomas Babington] Macaulay, poesía, pintar, exposiciones, conferencias, Hastings, Birmingham, Londres y Leam, y en general toda la belleza y las alegrías de la vida (GCPP Parkes 2/34). 
En las cartas que envió a Robert Fane y a su padre, Bessie articula su deseo de actuar en armonía con su concepto de sí misma. En ellas justifica su decisión de rechazar la propuesta de matrimonio alegando que disfrutaba del estilo de vida que tenía como joven soltera. También pone sobre la mesa lo que ella consideraba imprescindible antes de aceptar una proposición de matrimonio: que un hombre y una mujer se conocieran mutuamente en profundidad antes de casarse. La carta que le envía a su pretendiente es una respuesta "sencilla y directa" done le explica, cordial pero firmemente, que, a pesar de reconocer que se siente alagada y de estar convencida de "su carácter justo y de los méritos que creo que ha logrado", no puede satisfacer sus deseos: es decir, aceptar su petición de matrimonio.

La cordialidad con la que se dirige a su pretendiente se traduce, en la carta a su padre, en una carcajada ante "algo tan absurdo". Como explica en su carta a su padre, Bessie estaba perfectamente satisfecha disfrutando de las actividades que estimulaban su Bildung. Las palabras que concluyen su carta formal dirigida a Robert ("Créame, necesito más un profesor que un marido") reflejan sus prioridades: la realización intelectual y artística más que las obligaciones maritales. En la carta a su padre, esta realización personal se ve expresada de una forma más directa: "Me aferro a la satisfacción que me procura, querido Papá, lo que espero será, durante mucho tiempo, mi estilo de vida actual, con Mill, latín, montar a caballo, las críticas literarias, Macaulay, poesía, pintar, exposiciones, conferencias, Hastings, Birmingham, Londres y Leam, y en general toda la belleza y las alegrías de la vida”. De hecho, sabemos que Bessie escribió poemas desde muy joven, publicó algunos en la prensa local, The Birmingham Journal, y consiguió publicar una antología a los 23 años.

La conciencia feminista que parece apuntalar el posicionamiento que adopta Bessie se expresa de forma algo ambigua en su carta a Robert. Su discurso se sitúa entre la normatividad de género ("una mujer debe, en gran medida, dejarse absorber per la vida y los proyectos de su marido. ... Ella deberá ser su devota compañera, no sólo a través de su amor sino a través de su 
conciencia y su intelecto") y una afirmación que puede interpretarse como, o bien una descripción, o bien una crítica al contexto de la época ("en el estado actual de la sociedad”). Esta ambigüedad se resuelve en una carta que Bessie le escribió a Barbara sólo unos meses más tarde, donde da prioridad a su propia autonomía antes que a las obligaciones culturales y sociales:

Mi querida Amiga, me pregunto si algún día tendrás que arrastrarme para que me interese en los asuntos de amor. No te parece la mera mención completamente ridícula ... nuestras ideas sobre el amor y el matrimonio son tan particularmente diferentes a las actuales (GCPP Parkes 5/46).

En resumen, las cartas que Bessie envió a su pretendiente, a su padre y a Barbara representan su intento de ejercer su autonomía: en términos de Meyers, de actuar en armonía con su conciencia de sí misma. En ellas adopta un discurso individualizado que incorpora una perspectiva feminista sobre los derechos de la mujer, soltera y casada, a la realización personal ("nuestras ideas sobre el amor y el matrimonio son tan particularmente diferentes a las actuales”). Y esta combinación única incluye una aceptación ambigua en relación a ciertas expectativas de género tradicionales. Después de un intercambio de cartas a lo largo de unas semanas (algo resentido por parte de Robert ante el rechazo de Bessie, y algo ofendido y alertado el padre por el hecho de que su hija hubiera tomado la iniciativa de liderar la propuesta sin antes consultarle), la familia dio por concluido el affaire (GCPP Parkes 2/35, 2/36, 2/37, 2/38, 2/45, 9/110, 9/111, 9/112).

Un año más tarde, en 1850, Bessie entamó una correspondencia amical con un primo suyo lejano, Sam Blackwell, un forjador viudo. Había recientemente renovado la comunicación con él, y las cartas pronto se convirtieron en una correspondencia de noviazgo. Mantuvieron el compromiso de matrimonio durante 10 años, periodo que incluyó varias interrupciones (HIRSCH, 1998, 53, 99-101, 173, 234). Similar al caso de Robert Fane, durante su compromiso Bessie se sirvió de la correspondencia, con Sam, sus padres y 
amigas íntimas, para ejercer su autonomía.

A modo de ejemplo, a finales de julio de 1854, Bessie y Barbara alquilaron una casa de campo en el norte del País de Gales para pasar unos días de vacaciones. Allí, Barbara editó los últimos retoques de su panfleto Brief Summary y Bessie el de su ensayo Remarks on the Education of Girls (HIRSCH, 1998, 99). El 28 de julio esta última escribió a su prometido:

durante todos estos años desde 1847 hemos estado a penas solos para poder adquirir ese tipo de conocimiento confidencial de las complejidades del carácter que ocurre con amigos del mismo sexo. Tampoco me has visto entre mis amistades, con las que tengo total libertad de hablar y actuar. Mis hábitos como mujer independiente han sido durante años tan diferentes a los de la mayoría de las mujeres que me hacen reticente ante la idea de contribuir a la felicidad de otro y de mí misma en el marco del matrimonio. Siento que el interés genuino que tengo por la política, la literatura y la sociedad y en concreto en todo lo que concierne la posición legal y social de mi propio sexo, es tal que es raro que los hombres lo aprecien en una mujer. Esto me ha mantenido estos 25 años peculiarmente distante de la mayoría de ellos. Eso es lo que soy ahora y lo que quiero continuar siendo esencialmente. ... mis opiniones no son flexibles, y he iniciado una carrera literaria que es, para mí, el sentido de mi vida profesional. Estoy escribiendo como tú: con honestidad. Y si tú, que en el pasado disfrutaste de una vida matrimonial feliz, consideras que soy poco razonable en mi recelo sobre este tema, es porque quizás no sabes hasta qué punto las mujeres educadas y cultas están empezando a desconfiar del matrimonio tal y como es en la actualidad (GCPP Parkes 9/6).

Adoptando un discurso directo articulado desde una perspectiva feminista, en su carta Bessie le explica a Sam que del matrimonio ella esperaba amor mutuo, así como compatibilidad temperamental e intelectual. Con el fin de conocer íntimamente al hombre que pretendía casarse con ella, Bessie exigía un noviazgo de larga duración y con intercambios a solas. Pero, como explicaba también en otras cartas, deploraba la falta de interacción libre que sus padres y la sociedad le permitían (carta de Bessie a su amiga Kate Jevons, GCPP Parkes 6/62; carta de Bessie a Barbara, GCPP Parkes 5/36). 
Como he mencionado antes, durante 1854 Barbara estuvo recabando información sobre las leyes británicas relacionadas con el matrimonio y, durante su estadía en el País de Gales, se dedicó a editar y terminar su manuscrito, que publicó en otoño de ese año. Durante ese tiempo, Barbara discutió con Bessie la libertad legal que la mujer soltera disfrutaba y de la pérdida de personalidad legal que el contrato matrimonial súbitamente suponía. Como escribió de forma sucinta en Brief Summary: "Una mujer soltera tiene los mismos derechos que un hombre en términos de propiedad, protección de la ley, y pago de impuestos al Estado" (BODICHON, 1854, p. 23). Sin embargo, una vez casada, "Un hombre y una mujer son una sola persona ante la ley; la esposa pierde todos sus derechos como mujer soltera, y su existencia es completamente absorbida por la de su marido" (BODICHON, 1854, p. 25). Plenamente consciente de los riesgos que el hecho de pasar a ser una esposa implicaba, Bessie le explicó a Sam hasta qué punto se identificaba con esas "mujeres educadas y cultas" que, conscientes del sistema legal en vigor, eran reacias a comprometerse en el matrimonio.

Para asegurarse de que existía una buena congenialidad entre ellos y con el fin de evitar encuentros artificiales e incómodos durante las reuniones familiares, Bessie pidió a Sam que viniera a verla a Londres. Sugirió encontrarse allí, entre sus amigas, para que éste se hiciera una idea clara de su carácter y estilo de vida independientes, antes de que un compromiso matrimonial fuera concluido. Como le había explicado previamente a Robert, Bessie estaba dispuesta a asumir su rol de compañera para su futuro marido. Pero entendía este rol como uno que le aportara, simultáneamente, atención respecto a sus propias necesidades e intereses intelectuales. Bessie esperaba de su futuro marido el mismo respeto hacia su propia persona que ella estaba dispuesta a otorgar a la de su marido, incluido respecto por su "carrera literaria", que para ella representaba "el sentido de mi vida profesional".

No disponemos de las cartas que Sam le pudo contestar, pero, a judgar por las cartas que Bessie le envió y poniendo en práctica el concepto de 
relacionalidad narrativa, parece ser que éste, por lo menos en teoría, aprobaba el "descaro femenino juvenil" de Bessie. El 28 de septiembre de 1854 Bessie le escribió:

Estoy sumamente contenta de que apruebes mi "descaro femenino juvenil”. En efecto, hay muy poco de dama femenina en mí; no me gusta la palabra, y siempre me refiero a mí misma como a una mujer; tengo muy poca afinidad con la mayoría de damas ... Si me casara contigo, debería poner en tus manos toda mi vida; ... tengo tanto que perder, hay tantas cosas que un hombre puede destruir, tantos proyectos e intereses, amigos y esperanzas, que ninguna pasión puede reparar (GCPP Parkes 9/8).

La relación poco definida que Bessie y Sam tuvieron fue el objeto de comentarios en su entorno. Para evitar este cotilleo, la madre de Bessie, Elizabeth Parkes, hizo presión para que la pareja se comprometiera oficialmente antes de que los dos jóvenes pudieran encontrarse bajo las condiciones de intimidad y libertad que pedía su hija (carta de Bessie a Barbara, GCPP 5/44). El razonamiento epistolar de Bessie en la carta que le escribió a su madre el 15 de diciembre respecto a esta posibilidad, revela un punto de vista feminista perspicaz:

Aunque he intentado aceptar la idea de un compromiso inmediato para poder satisfacer lo que parecen ser tus sentimientos, soy incapaz de hacerlo; ... Parece ser que piensas que en un compromiso formal y en una boda inmediata encontraré una cierta seguridad. Sólo lo sería en el caso de que yo fuera feliz en ese compromiso y en ese matrimonio. $\mathrm{Si}$, viéndonos con libertad, él o yo encontráramos que existen discrepancias, pondría con total certeza punto y final a cualquier compromiso, por muy público que fuera, antes de sacrificar toda una vida. De la misma manera, si, después de casados, nos diéramos cuenta de que somos incompatibles, ninguno de los dos seríamos nadie para arruinar toda una vida con una unión sólo por el bien de la opinión pública ... toda la estima que le tengo hacia su persona y la atracción que confieso que siento por él no hacen que me precipite a comprometerme en una unión que implica tanta responsabilidad, cuyas leyes y costumbres, ya lo sabes tú bien, discrepo frontalmente. Es inútil y cruel que discutamos sobre este 
tema, como si yo fuera una mujer normal. Sabes, mi querida Madre, que mis opiniones y mi estilo de vida se asientan profundamente en mis inquietudes intelectuales y en mis proyectos personales. La vida de casada no respetará fácilmente mis necesidades como le pasa a la mayoría de mujeres ... Por favor, enséñale a mi Padre esta carta. Me he expresado con toda la claridad y simplicidad de la que soy capaz, después de 9 semanas de reflexión (GCPP Parkes 2/4).

A lo largo de su vida, Bessie fue una hija respetuosa y agradecida para con el cariño y atención que recibió de sus padres, incluidos los consejos con los que no estaba de acuerdo; pero también tuvo una fuerte conciencia de sí misma y expresó sin rodeos su determinación. Los fragmentos de carta que he citado testimonian de su intento de actuar de forma autónoma. Representan cómo negocia su deseo de actuar independientemente de la opinión de su pretendiente, sus padres o la sociedad. Y su discurso tiene una base feminista. Identificándose con aquellas "mujeres educadas y cultas" que adoptaban un discurso feminista, como su amiga Barbara, por ejemplo, y distanciándose de las "damas", que representaban la normatividad de género, Bessie adopta un discurso como joven casadera que exige, por un lado, su derecho a entrar en un compromiso según sus propios términos y, por otro lado, su derecho a la realización personal en el seno de una futura unión matrimonial. Consciente de las "leyes y costumbres" que hacen temblar a las "mujeres educadas y cultas" (el tipo de pérdida legal que Barbara señalaba en su Brief Summary), Bessie pactó su noviazgo y su futura vida matrimonial en sus cartas a Sam, a su madre y, a través de ésta, a su padre. Incluso sugería la posibilidad de romper su compromiso y de separarse de su esposo en el caso de que hubieran "discrepancias" e incompatibilidades a nivel temperamental e intelectual. Con el acto de priorizar su felicidad individual sobre las costumbres y obligaciones sociales, en su razonamiento epistolar Bessie desafío directamente la normatividad de género.

$\mathrm{Al}$ cabo de 10 años Bessie y Sam rompieron su ambiguo noviazgo. Bessie decidió casarse, a la edad de 38 años, con el hombre que ella misma eligió: un pintor francés, Louis Belloc, que conoció durante una estadía en París con 
Barbara en 1867. Con él tuvo dos hijos y, en razón de la invalidez de su marido, enviudó a los pocos años de estar casada.

No sabemos si Anna Mary y Barbara recibieron cartas con proposiciones de matrimonio. Si las recibieron, tampoco sabemos bajo qué condiciones ejercieron su razonamiento epistolar, ni que forma tuvo éste. Sin embargo, podemos sugerir que es posible que, por carta o de viva voz, estas dos amigas ejercieran, de una forma similar, su autonomía como mujeres casaderas en relación a sus sentimientos amorosos, su noviazgo y su futura vida de esposas.

De Barbara sabemos, de su propia voz epistolar, que durante su adolescencia y juventud disfrutó de las actividades que estimulaban su Bildung (GCPP Parkes 4 y 5). Como Bessie, empezó a publicar sus primeros artículos de prensa a los 21 años en el periódico local, Hastings and St Leonards News: en defensa de la salud pública, de la educación de las mujeres, y en pro de unos códigos vestimentarios para las mujeres que fueran cómodos para un estilo de vida activo. Además, como he explicado en otras publicaciones, Barbara convino por carta las condiciones de su vida matrimonial una vez casada en diálogo con su familia (SIMON-MARTIN, 2020). Podemos suponer que también lo hizo en relación con los pretendientes que rechazó. De hecho, Barbara no aceptó casarse hasta que no dio con el hombre de sus sueños, el Dr. Eugène Bodichon; y tomó la decisión de casarse con él, bien a disgusto de su familia, que consideraba que un francés, 17 años mayor que ella, no era el marido ideal. En efecto, sabemos que, una vez casada, a fin de poder lograr el estilo de vida independiente en el seno de la vida matrimonial que proyectó en sus publicaciones (a fin de poder actuar en armonía con el concepto que tenía de sí misma), en el marco de su vida "nómada", Barbara articuló una redefinición de las creencias de género respecto al matrimonio en sus cartas enviadas a su familia, ya como mujer casada. Y es que las expectativas culturales que imponían a la mujer como esposa y madre en relación a sus obligaciones estaban en tensión directa con el tipo de proyectos personales que vervalizó en sus artículos y panfletos, como por 
ejemplo en Women and Work (1857). Expresados y circulados en textos médicos, libros de consejos, revistas para mujeres, sermones religiosos y discursos políticos, la domesticidad burguesa requería el sacrificio de la individualidad de la mujer. Estas expectativas de género enfatizaban una naturaleza distintamente femenina - atenta para con las necesidades de los demás, intuitiva, amable, paciente, sacrificada - para justificar sus obligaciones domésticas y maternales. Esta diferenciación sexual iba en detrimento de sus propios intereses y talentos. Y es que se esperaba de las mujeres, ya sea casadas o solteras, que contribuyeran a crear un contexto doméstico favorable para prestar asistencia y nutrir los intereses, ambiciones y deseos de los miembros masculinos del hogar - una visión que fue famosamente plasmada en el exitoso libro de John Ruskin Sesame and Lilies (1865).

De Anna Mary tampoco tenemos información directa para apoyar con absoluta certeza la hipótesis de que, como Bessie, ejerció su razonamiento epistolar con sus pretendientes. Sabemos que se escribió cartas con Edward Bateman durante su estancia en Múnich, pero ninguna carta intercambiada con él o Alfred Watts ha sobrevivido. Lo que sí sabemos es que decidió formarse en la Henry Sass' Art Academy y posponer su matrimonio con Bateman para poder estudiar con von Kaulbach durante dos años, lo que nos da una idea de cuáles eran sus prioridades a la edad de 20-25 años. De hecho, su voz epistolar en las cartas que se intercambió con Barbara durante su formación en Múnich, nos da una idea de cómo se proyectaba como estudiante de arte (que articuló en su libro An Art-Student in Munich, 1853), y como futura Señora Bateman (Columbia University, Butler Library, Leonore Beaky, Letters of Anna Mary to Barbara). En estas cartas, Anna Mary, sin disimular la ilusión que le hacía ser la esposa de Edward Bateman, también expresó cuál era su prioridad: su realización profesional artística, no sólo como estudiante de arte comprometida con Edward Bateman, sino como futura profesora de arte para mujeres artistas ya como esposa de éste. 


\section{CONCLUSIÓN}

En el marco de una lectura de la carta como fuente de Bildung, en este artículo he examinado el razonamiento epistolar (término acuñado por la historiadora Dena Goodman) de Anna Mary Howitt, Barbara Bodichon y Bessie Parkes en relación con una dimensión concreta del desarrollo personal: el amor y el matrimonio. Dado que los archivos personales de estas tres amigas son limitados, de forma paralela, he hecho una pequeña reflexión epistemológica sobre la carta como fuente de conocimiento histórico. Basándome sobre todo en la voz epistolar de Bessie, cuyo archivo personal es el más extenso, he sugerido e ilustrado con fragmentos de cartas que los diálogos epistolares sirvieron de espacio donde las tres amigas negociaron y ejercieron su autonomía (entendida aquí bajo el prisma del proyecto teórico de la filósofa feminista Diana Meyers) y, de forma concomitante, donde articularon una redefinición de los conceptos de feminidad, amor y matrimonio. Recurriendo al concepto de relacionalidad narrativa de Sidonie Smith y Julia Watson he sugerido que el silencio del "yo" epistolar de Barbara en una conversación con Bessie y Anna Mary sobre el poema de Alfred Tennyson The Princess puede ser "colmado" si recurrimos a su "tú" epistolar, tal y como se proyecta en las cartas que sus dos amigas le enviaron. Además, el "ella" epistolar de Anna Mary en las cartas que Bessie le escribió a Barbara, nos permite enriquecer a su vez el análisis del razonamiento epistolar en la conversación que he reproducido en la primera sección. En la segunda sección, he recurrido a la hipótesis para hacer frente al silencio archivístico de Anna Mary y Barbara respecto a la negociación y ejercicio de su autonomía como mujeres casaderas. Tomando el razonamiento epistolar de Bessie con sus pretendientes, familiares y amigas como referente y teniendo en cuenta la correspondencia personal de Anna Mary y Barbara en su conjunto, he sugerido que podemos hipotetizar sobre la posibilidad de que estas dos amigas pactaran y ejercieran su autonomía de una forma similar. 


\section{REFERENCIAS}

BLEICHER, Josef. Bildung. Theory Culture Society, v. 23, p. 364-365, 2006. BODICHON, Barbara. A Brief Summary, in Plain Language, of the Most Important Laws Concerning Women. Londres: John Chapman, 1854. In: LACEY, Candida Ann (Coord.). Barbara Leigh Smith Bodichon and the Langham Place Group. London: Routledge, 2001.

BODICHON, Barbara. Women and Work. Londres: John Chapman, 1857.

BRUFORD, Walter Horace. The German Tradition of Self-Cultivation: 'Bildung' from Humboldt to Thomas Mann. Cambridge: Cambridge University Press, 1975.

GLEADLE, Kathlyn. The Early Feminists: Radical Unitarians and the Emergence of the Women's Movement, 1831-1851. Basingstoke: Palgrave Macmillan, 1995.

GOODMAN, Dena. Becoming a Woman in the Age of Letters. Ithaca: Cornell University Press, 2009.

HIRSCH, Pam. Barbara Bodichon: Feminist, Artist and Rebel. London: Chatto and Windus, 1998.

HOWITT, Anna Mary, An Art-Student in Munich. 1853.

HUMBOLDT, Wilhelm von, The Eighteenth Century. 1796-1797.

HUMBOLDT, Wilhelm von. Theorie der Bildung des Menschen. c.17931794.

LØVLIE, Lars; MORTENSEN Klaus; NORDENBO Sven (Coords.). Educating Humanity: Bildung in Postmodernity. Oxford: Blackwell, 2003.

LÜTH, Christoph. On Wilhelm von Humboldt's Theory of Bildung Dedicated to Wolfgang Klafki for his $70^{\text {th }}$ Birthday. Journal of Curriculum Studies, vol. 30, n. 1, p. 43-60, 1998.

MEYERS, Diana T. Self, Society and Personal Choice. New York and Oxford: Columbia University Press, 1989.

PARKES, Bessie. Remarks on the Education of Girls. Londres: [desconocido], 1854.

RENDALL, Jane. The Origins of Modern Feminism. Women in Britain, 
France and the United States, 1780-1860. Basingstoke: Macmillan, 1985.

RUSKIN, John. Sesame and Lilies. London: Smith, Elder and Co., 1865.

SHATTOCK, Joanne. Elizabeth Rayner Parkes Belloc. Oxford Dictionary of National Biographies. Available

at:https://www.oxforddnb.com/view/10.1093/ref:odnb/9780198614128.001.o o01/odnb-9780198614128-e-41193 Accedido el: 26 noviembre, 2019.

SIMON-MARTIN, Meritxell. Barbara Leigh Smith Bodichon's Bildung:

Education, Feminism and Agency in Letters. In: BUMBARIS, Alexia et al. (Coords.). Frauen- und Geschlechtergeschichte un/diszipliniert? Aktuelle Beiträge aus der jungen Forschung. Innsbruck: StudienVerlag, 2016, p. 41-65.

SIMON-MARTIN, Meritxell. Barbara Bodichon's Travel Writing: Her Epistolary Articulation of Bildung. History of Education, vol. 45, n. 3, p. 285-303, 2016a.

SIMON-MARTIN, Meritxell. Educational Place and Space: The Unconventional Education of Barbara Leigh Smith Bodichon (1827-1891). History of Education Researcher, mayo, n. 89, p. 7-17, 2012.

SIMON-MARTIN, Meritxell. Barbara Bodichon's Epistolary Education: Unfolding Feminism. London: Palgrave Macmillan, No prelo 2020.

SIMON-MARTIN, Meritxell. Barbara Bodichon's Archive: Silences that Speak. No prelo 2020a.

SMITH, Sidonie; WATSON, Julia. Reading Autobiography: A Guide for Interpreting Life Narratives. Minneapolis: University of Minnesota Press, 2001. TENNYSON, Alfred. The Princess. A Medley. London: Edward Moxon, 1847.

VARKØY, Øyvind. The Concept of Bildung. Philosophy of Music Education Review, v. 18, n. 1, p. 85-96, 2010.

WIMMER, Michael. Ruins of Bildung in a Knowledge Society: Commenting on the Debate about the Future of Bildung. Educational Philosophy and Theory, vol. 23, n. 2, p. 167-187, 2003.

WULF, Christoph. Perfecting the Individual: Wilhelm von Humboldt's Concept of Anthropology, Bildung and Mimesis. Educational Philosophy and Theory, vol. 35, n. 2, p. 241-249, 2003. 
MERITXELL SIMON-MARTIN is Marie Curie Fellow at Roehampton University. Her areas of research interest lie at the crossroads of History of Education, Women's and Gender History, Narrative Analysis and Epistolary Studies. She has published extensively on Barbara Bodichon, the history of feminism in Europe and on epistolarity. She is co-editor of the History of Education Researcher (History of Education Society UK) and member of the editorial board of the European Journal of Life Writing (International Auto/biographical Association). She runs the research blog Epistolarity:

E-mail: meritxellsimonmartin@gmail.com

(iD) http://orcid.org/0000-0002-9486-3020/

Recebido em: 15 de agosto de 2018

Aprovado em: 05 de janeiro de 2019

Revista História da Educação - RHE

Associação Sul-Rio-Grandense de Pesquisadores em História da Educação - Asphe Artigo de acesso aberto distribuído nos termos de licença Creative Commons. 